\title{
Voltammetric Determination of Dinitronaphthalenes Using a Silver Solid Amalgam Paste Electrode
}

\author{
Jana TVRDIKova, Ales DANHEL, ${ }^{\dagger}$ Vlastimil VysKociL, and Jiri BAREK ${ }^{\dagger}$ \\ Department of Analytical Chemistry, Faculty of Science, Charles University in Prague, UNESCO Laboratory of \\ Environmental Electrochemistry, Hlavova 8, Prague 2, 12843, Czech Republic
}

\begin{abstract}
The electrochemical behavior and application of a new sensor, a silver solid amalgam paste electrode (AgSA-PE), based on the mixture of a fine silver solid amalgam powder $\left(60: 40\left(\mathrm{w}_{\mathrm{Hg}} / \mathrm{w}_{\mathrm{Ag}}\right)\right)$ and a suitable organic pasting liquid (Paraffin oil) in a ratio of 20:1 (w/w), was investigated in an aqueous-methanolic media (1:1). This alternative working electrode provides simple preparation and handling, adequate mechanical stability, easily renewable electrode surface, sufficiently wide cathodic potential window (up to $-1200 \mathrm{mV}$ within a $\mathrm{pH}$ range of $2.7-12.3$ ), and sufficient sensitivity without any necessary pretreatment. The practical usability of the AgSA-PE was verified by the development of voltammetric methods for the determination of selected environmentally important pollutants (1,3-, 1,5-, and 1,8-dinitronaphthalenes) in an aqueous-methanolic media (1:1). The differential pulse voltammetric methods at AgSA-PE give linear concentration dependences in the range of $1-100 \mu \mathrm{mol} \mathrm{l}^{-1}$ with limits of detection of about $1 \mu \mathrm{mol} \mathrm{l^{-1 }}$ in a mixture of Britton-Robinson buffer of appropriate $\mathrm{pH}$ and methanol (1:1).
\end{abstract}

(Received October 10, 2011; Accepted February 20, 2012; Published April 10, 2012)

\section{Introduction}

Mercury electrodes still represent a useful tool in the electroanalysis of various electrochemically reducible organic compounds, ${ }^{1} e . g$. in proteomic studies, for which they may offer a wide cathodic potential window, easily renewable electrode surface and high sensitivity. ${ }^{2,3}$ However, legislative restrictions of mercury use and ecological requirements for new electrochemical detectors have initiated the development of novel electrode materials, such as metal-mercury amalgams, ${ }^{4}$ composite $^{5}$ and/or carbon based materials. Carbon-based electrodes, such as a glassy carbon, boron-doped diamond and/or carbon-paste electrodes (CPE), ${ }^{7,8}$ are mostly applied for anodic measurements, and nowadays mainly used for the preparation of various specific biosensors..$^{9,10}$

Modern electroanalytical applications demand more sensitive, selective, robust and mechanically stable sensors. According to our opinion, a silver solid amalgam belongs to that of the most useful modern electrode materials, thanks to its simple preparation, good mechanical stability, wide cathodic potential window comparable with mercury electrodes and sufficient sensitivity in organic electroanalysis. This fact may be confirmed by the number of applications that have already been developed and rewieved. ${ }^{11-13}$ Passivation of the electrode surface is the main disadvantage of solid electrodes, which is commonly solved by their time-consuming mechanical and/or electrochemical pretreatment. Therefore, the ideas of the CPE with a simply renewable electrode surface and a silver solid amalgam with high hydrogen over-potential were combined. A novel paste electrode material based on a mixture of the silver

† To whom correspondence should be addressed.

E-mail: danhel.ales@gmail.com; barek@natur.cuni.cz amalgam powder and a proper pasting liquid was developed. It has already been shown that fine powder of silver solid amalgam mixed with Paraffin oil (or possibly with some other suitable pasting liquids) in a proper weight ratio of 20:1 (w/w) represents a novel paste electrode material suitable for voltammetric measurements in aqueous media. This silver solid amalgam paste electrode (AgSA-PE) was used for the voltammetric determination of 4-nitrophenol as a model compound in the aqueous media, ${ }^{14}$ and it was proved that its sufficiently wide potential window and easily renewable electrode surface provides a new and promising alternative in organic electrochemistry without any necessary time-consuming pretreatment. To verify the usability of this non-traditional electrode material in mixed aqueous-methanolic media, commonly used for less soluble organic compounds, is the main aim of this work. For this purpose, well electrochemically studied environmentally important and genotoxic pollutants (1,3-, 1,5- and 1,8-dinitronaphthalenes), ${ }^{15-18}$ were chosen as the model compounds, and methods for their voltammetric determination were developed.

\section{Experimental}

\section{Reagents and chemicals}

A silver solid amalgam was prepared by mixing of mercury (99.999\%, Polarografie Praha, Czech Republic) and silver powder $(2-3.5 \mu \mathrm{m}, 99.9 \%$, Sigma-Aldrich, Germany) in a weight ratio $\left(60: 40, \mathrm{w}_{\mathrm{Hg}} / \mathrm{w}_{\mathrm{Ag}}\right)$ by $1 \mathrm{~min}$ shaking on a Vortex Genie 2 (Scientific Industries, USA) in a 2-ml Eppendorf tube. The thus-prepared solid amalgam was then immediately pulverized to a rough powder in an agate mortar, and left to harden overnight. Afterwards, the rough amalgam powder was pulverized to a fine amalgam powder in the same agate mortar. 
Paraffin oil (Paraffinum liquidum, Pharmacopoeia Bohemia, Czech Republic) was used for the preparation of amalgam pastes in an experimentally optimized mass ratio of 20:1 (amalgam:Paraffin oil). The thus-prepared silver solid amalgam paste is stable for at least 6 months with no significant changes in its electrochemical behavior.

The investigated analytes (1,3-dinitronaphthalene (97\%, 1,3-DNN), 1,5-dinitronaphthalene (98\%, 1,5-DNN), and 1,8-dinitronaphthalene $(97 \%, 1,8$-DNN)) were all purchased from Sigma-Aldrich, Germany, and their $1 \times 10^{-3} \mathrm{~mol} \mathrm{l}^{-1}$ stock solutions were prepared in methanol ( $>99.9 \%$, Merck, Germany). Boric acid, acetic acid (99.8\%), phosphoric acid (85\%) and sodium hydroxide (all p.a. purity, Lach-Ner, Czech Republic) were used for the preparation of Britton-Robinson (BR) buffers by mixing a $0.2 \mathrm{~mol} \mathrm{l}^{-1} \mathrm{NaOH}$ solution with a solution containing $0.04 \mathrm{~mol} \mathrm{l}^{-1}$ of the acids mentioned above. Oxygen was removed from the measured solutions by 5 min of deaeration with nitrogen of 4.0 purity (Linde Praha, Prague, Czech Republic). All experiments and measurements were conducted at laboratory temperature.

\section{Apparatus}

Voltammetric measurements were carried out by an Eco-Tribo Polarograph operated by software PolarPro Ver. 5.1 (both Polaro-Sensors Praha, Czech Republic) under a Windows XP system (Microsoft, USA) using a three-electrode system in a 10-ml glass vessel. The Teflon body of the paste electrode (inside diameter, $1.70 \mathrm{~mm}$ ) was stuffed with amalgam paste, and used as the working electrode. A silver chloride reference electrode ( $\mathrm{Ag} \mid \mathrm{AgCl}, 3 \mathrm{M} \mathrm{KCl}$, Type 10-20+, Electrochemical Detectors Turnov, Czech Republic) and a platinum wire auxiliary electrode (Monocrystals Turnov, Czech Republic) were used.

Direct current voltammetry (DCV) and cyclic voltammetry (CV) were used at a scan rate of $20 \mathrm{mV} \mathrm{s}^{-1}$ and the same scan rate with a pulse height of $-50 \mathrm{mV}$ and a pulse width of $100 \mathrm{~ms}$ was used in differential pulse voltammetry (DPV) and adsorptive stripping differential pulse voltammetry (AdSDPV).

Exact $\mathrm{pH}$ values of the prepared BR buffers and the experimental $\mathrm{pH}\left(\mathrm{pH}^{\mathrm{exp}}\right)$ values of $\mathrm{BR}$ buffer-methanol mixtures (1:1) were measured by a Jenway, UK, digital $\mathrm{pH}$-meter (Type 3510) using a combined glass electrode calibrated with standard aqueous buffers at laboratory temperature.

\section{Procedures}

The solutions for voltammetric measurements were prepared by mixing $5 \mathrm{ml}$ of a BR buffer of selected $\mathrm{pH}$ with about $3 \mathrm{ml}$ of methanol and a proper volume of dinitronaphthalene (DNN) stock solution and filled by methanol to the mark of a $10-\mathrm{ml}$ volumetric flask. The $\mathrm{pH}^{\exp }$ of the thus-prepared supporting electrolytes composed from BR buffer and methanol (1:1) were measured by a $\mathrm{pH}$-meter. The analyzed solutions were deaerated for $5 \mathrm{~min}$ with nitrogen before measurements. Prior to entering the voltammetric glass vessel, the nitrogen was passed through a gas-washing bottle containing a deionized water-methanol mixture (1:1) (i.e. the same volume ratio as in analyzed solution). Voltammograms were registered in selected potential ranges using the parameters of the used methods as described above.

The peak heights $\left(I_{\mathrm{p}}\right)$ of the studied DNNs were evaluated from a manually fitted baseline. Wave heights obtained by $\mathrm{CV}$ were evaluated as the distance of two straight lines, constructed from linear increasing/decreasing parts before and after increase/decrease of the sigmoid waves, at potentials corresponding to the found inflexion points.
Table 1 Potential ranges applicable for DCV and DPV measurements at AgSA-PE in BR buffer-methanol (1:1) with appropriate $\mathrm{pH}^{\text {exp }}$

\begin{tabular}{|c|c|c|c|c|c|}
\hline \multicolumn{2}{|c|}{ Method } & \multicolumn{2}{|c|}{ DCV } & \multicolumn{2}{|c|}{ DPV } \\
\hline $\begin{array}{c}\text { BR buffer } \\
\text { pH }\end{array}$ & $\mathrm{pH}^{\exp }$ & $E_{\text {in }} / \mathrm{mV}$ & $E_{\text {fin }} / \mathrm{mV}$ & $E_{\text {in }} / \mathrm{mV}$ & $E_{\mathrm{fin}} / \mathrm{mV}$ \\
\hline 2.0 & 2.7 & 60 & -1260 & -100 & -1100 \\
\hline 3.0 & 3.9 & 150 & -1220 & -100 & -1100 \\
\hline 4.0 & 4.6 & 150 & -1260 & -100 & -1200 \\
\hline 5.0 & 5.5 & 130 & -1220 & -100 & -1200 \\
\hline 6.0 & 6.9 & 80 & -1220 & -100 & -1150 \\
\hline 7.0 & 8.2 & 90 & -1200 & -100 & -1200 \\
\hline 8.0 & 8.7 & 60 & -1200 & -100 & -1150 \\
\hline 9.0 & 9.5 & 60 & -1200 & -100 & -1100 \\
\hline 10.0 & 10.5 & 30 & -1200 & -200 & -1100 \\
\hline 11.0 & 11.7 & 20 & -1200 & -250 & -1100 \\
\hline 12.0 & 12.3 & -70 & -1200 & -250 & -1100 \\
\hline
\end{tabular}

$\mathrm{pH}^{\mathrm{exp}}$, experimental $\mathrm{pH}$ of $\mathrm{BR}$ buffer-methanol mixture (1:1) measured by combined $\mathrm{pH}$ glass electrode calibrated on standard aqueous buffers.

$E_{\text {in }}$, initial potential (beginning of silver amalgam oxidation).

$E_{\text {fin }}$, final potential (beginning of oxygen reduction).

\section{Results and Discussion}

The paste electrode material composed from the silver solid amalgam fine powder $\left(60: 40, \mathrm{w}_{\mathrm{Hg}} / \mathrm{w}_{\mathrm{Ag}}\right)$ and the Paraffin oil (pasting liquid) mixed in mass ratio 20:1 (w/w) was found as the most suitable in a previous study, ${ }^{14}$ and was used in this work. This electrode material, previously studied in aqueous media (0.1 $\mathrm{mol} \mathrm{l}^{-1} \mathrm{HClO}_{4}, 0.1 \mathrm{~mol} \mathrm{l}^{-1} \mathrm{HCl}, 0.2 \mathrm{~mol} \mathrm{l}^{-1}$ acetate buffer pH 4.8, $\quad 0.05 \mathrm{~mol} \mathrm{l}^{-1} \mathrm{Na}_{2}$ EDTA $+0.2 \mathrm{~mol} \mathrm{l}^{-1}$ acetate buffer $\mathrm{pH} 4.8,0.1 \mathrm{~mol} \mathrm{l}^{-1} \mathrm{NaClO}_{4}, 0.05 \mathrm{~mol} \mathrm{l}^{-1} \mathrm{Na}_{2} \mathrm{~B}_{4} \mathrm{O}_{7} \mathrm{pH} 9.2$, or $0.1 \mathrm{~mol} \mathrm{l}^{-1} \mathrm{NaOH}$ ), offers the widest cathodic potential window of up to $-1.0,-1.1$ and $-1.2 \mathrm{~V}$ in the acidic, neutral and alkaline aqueous media, respectively. The lowest background current and the highest signals of a testing compound (4-nitrophenol) were also observed for this paste electrode material. Commonly used CPEs may be theoretically used for cathodic measurements of up to $-1.3 \mathrm{~V}$ in neutral media. However, oxygen dissolved in the carbon paste gives mostly an unpredictable and unrepeatable broad signal at potentials higher than $-0.5 \mathrm{~V}$ which may interfere with the investigated signals up to hydrogen evolution.,19 However, the oxygen signal was not observed up to $-1.2 \mathrm{~V}$ at AgSA-PE utilizing the silver solid amalgam paste in neither the aqueous nor aqueous-methanolic media (1:1) studied in this work. Potential windows applicable for voltammetric measurements using the AgSA-PE were determined using DCV and DPV in the BR buffer-methanol mixture (1:1). Negative edges of the potential windows were found to be about $-1.2 \mathrm{~V}$ using DCV within the entire investigated $\mathrm{pH}^{\exp }$ range of $2.7-12.3$. The usable potential ranges are summarized in Table 1. The presence of methanol had no significant negative effects on the mechanical and electrochemical behavior of the amalgam paste, such as dissolution of the pasting liquid in the electrolyte, penetration of the methanol into the paste, paste stability in the mixed methanolic solutions, etc.

The applicability of the AgSA-PE using "Paraffin paste" (20:1) in the BR buffer-methanol (1:1) solution was further tested for the voltammetric determination of selected nitro-compounds: 1,3-, 1,5- and 1,8-DNN. This methanol/water ratio was based on our previous results of the polarographic and 
(A)
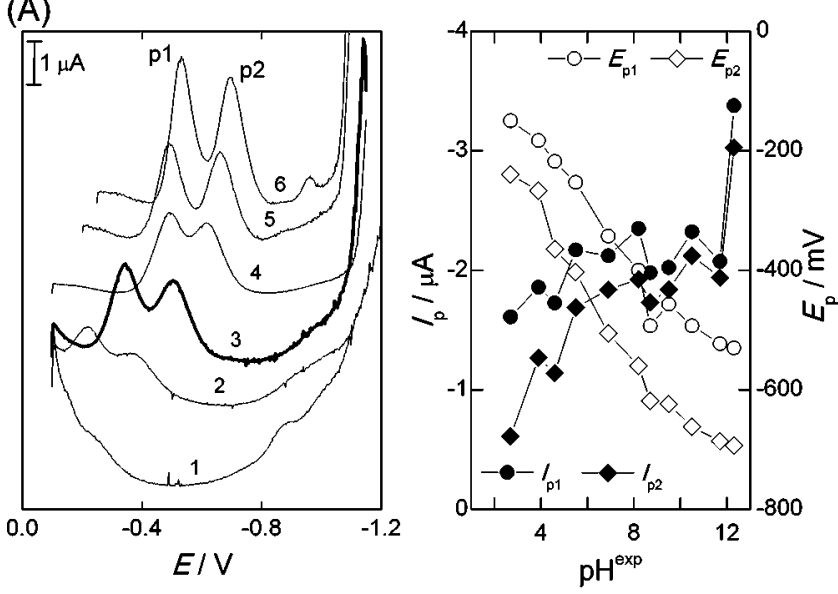

(B)
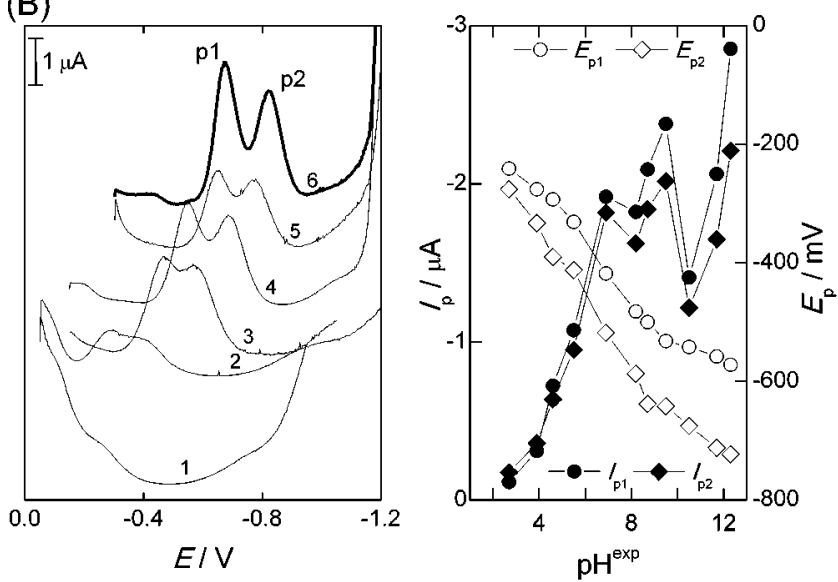

(C)
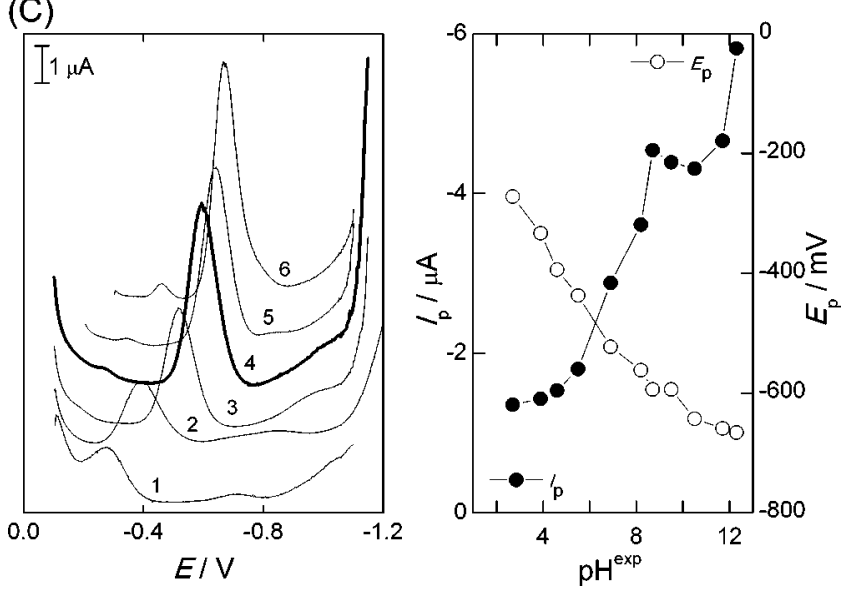

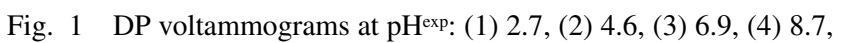
(5) 10.5 , (6) 12.3 of BR buffer-methanol mixture (1:1) and appropriate $\mathrm{pH}$ dependences of peak height $(\mathrm{s})\left(I_{\mathrm{p}}\right)$ and potential $(\mathrm{s})\left(E_{\mathrm{p}}\right)$ of: $(\mathrm{A})$ 1,3-DNN，(B) 1,5-DNN，(C) 1,8-DNN (all $c=1 \times 10^{-4} \mathrm{~mol} \mathrm{1}^{-1}$ ) measured at AgSA-PE. Scan rate, $20 \mathrm{mV} \mathrm{s}^{-1}, E v s$. $\mathrm{Ag} \mid \mathrm{AgCl}(3 \mathrm{M})$. Bold lines correspond to optimal $\mathrm{pH}^{\exp }$ of supporting electrolyte chosen for further studies.

voltammetric determination of the DNNs at mercury electrodes and on their limited solubility in water. The position of the electrochemically reducible nitro-groups on the aromatic system of naphthalene influences both the physical and chemical properties and the electrochemical behavior of model analytes. DP voltammograms of the DNNs with the dependencies of the
(A)
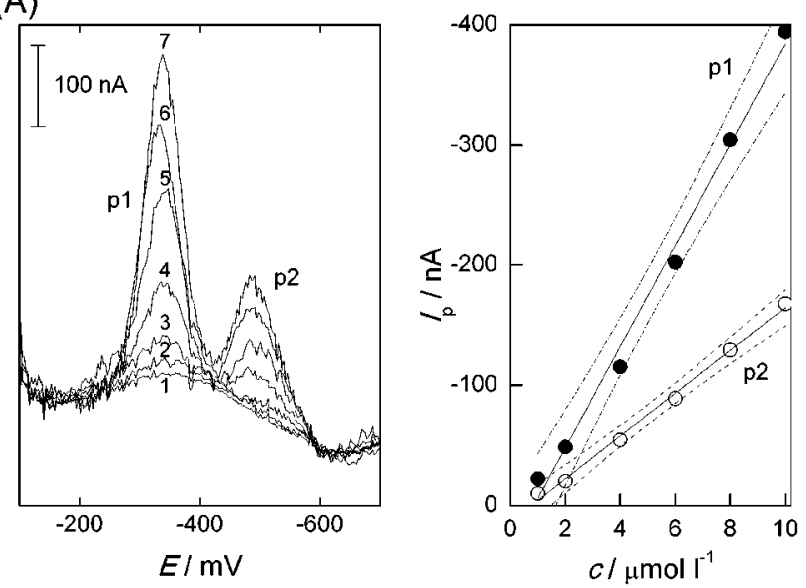

(B)
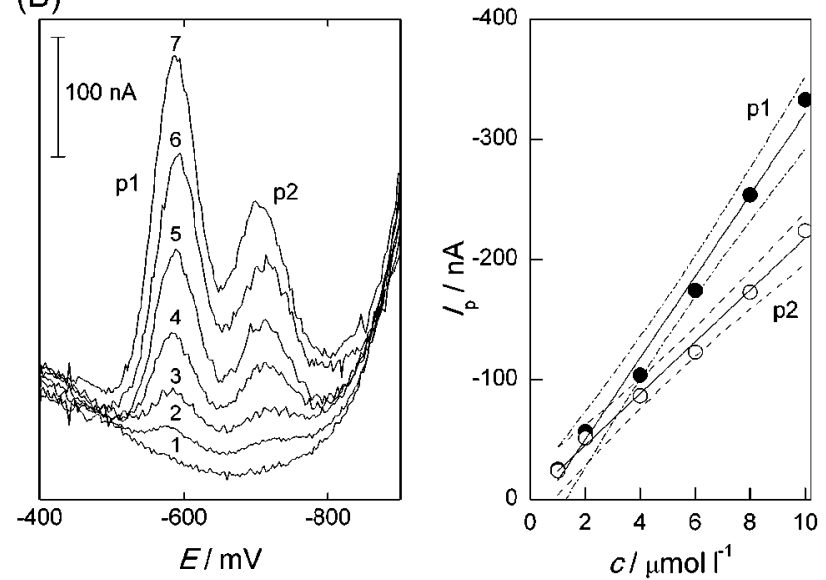

(C)
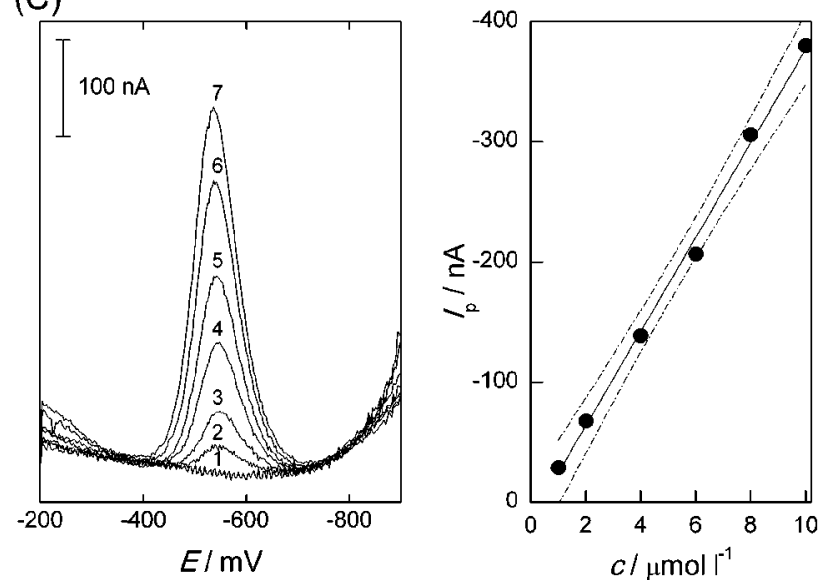

Fig. 2 DP voltammograms of: (A) 1,3-DNN, (B) 1,5-DNN, (C) 1,8-DNN at AgSA-PE in dependences on concentration $\left(c, \mu \mathrm{mol} \mathrm{l}^{-1}\right)$ : (1) 0 base electrolyte, (2) 1.0, (3) 2.0, (4) 4.0, (5) 6.0, (6) 8.0, (7) 10.0, measured in a BR buffer-methanol mixture (1:1) with appropriate $\mathrm{pH}^{\exp }$ : (A) 6.9 , (B) 12.3 , (C) 8.7, and evaluated calibration straight lines (solid lines) of first (p1) and second (p2) peaks with depicted confidence bands (confidence level 95\%, dash dot or dash lines); scan rate, $20 \mathrm{mV} \mathrm{s}^{-1}, E v s . \mathrm{Ag} \mid \mathrm{AgCl}(3 \mathrm{M})$.

evaluated peak heights $\left(I_{\mathrm{p}}\right)$ and peak potentials $\left(E_{\mathrm{p}}\right)$ on the $\mathrm{pH}^{\exp }$ are depicted in Fig. 1. It is obvious that the peak potentials, and mostly also the peak heights of the DNNs increase with increasing $\mathrm{pH}^{\text {exp }}$. The 1,3-, 1,5-, and 1,8-DNN give two, two and one DPV peaks in the entire studied $\mathrm{pH}$ range, respectively, 
Table 2 Signal stability of studied DNNs $\left(c=1 \times 10^{-4} \mathrm{~mol} \mathrm{l}^{-1}\right)$ determined by DPV at AgSA-PE in BR buffer-methanol (1:1) at selected $\mathrm{pH}^{\exp }$

\begin{tabular}{cccccrr}
\hline $\begin{array}{c}\text { Compound } \\
(\text { BR-methanol) }\end{array}$ & Peak & $\begin{array}{c}\text { No. of } \\
\text { series }\end{array}$ & $\begin{array}{c}\text { No. of } \\
\text { repetitions }\end{array}$ & $\begin{array}{c}\text { Average/ } \\
\text { nA }\end{array}$ & $\begin{array}{c}\text { SD/ } \\
\text { nA }\end{array}$ & $\begin{array}{c}\text { RSD } \\
\%\end{array}$ \\
\hline 1,3-DNN & 1 & 1 & 18 & -1999 & 66 & 3 \\
$\left(\mathrm{pH}^{\exp } 6.9\right)$ & 2 & 1 & $18(17)^{\mathrm{a}}$ & -1537 & 80 & 5 \\
& 1 & 7 & 5 & -1549 & 326 & 21 \\
& 2 & 7 & $5(4)^{\mathrm{a}}$ & -1378 & 255 & 19 \\
$1,5-\mathrm{DNN}$ & 1 & 1 & 18 & -2733 & 24 & 1 \\
$(\mathrm{pH} \exp 12.3)$ & 2 & 1 & $18(17)^{\mathrm{a}}$ & -2429 & 104 & 4 \\
& 1 & $7(6)^{\mathrm{a}}$ & 5 & -1189 & 113 & 10 \\
& 2 & 7 & $5(4)^{\mathrm{a}}$ & -861 & 71 & 8 \\
$1,8-\mathrm{DNN}$ & 1 & 1 & $18(17)^{\mathrm{a}}$ & -2833 & 8 & 0.3 \\
$\left(\mathrm{pH}{ }^{\exp 8.7)}\right.$ & 1 & 7 & $5(4)^{\mathrm{a}}$ & -3731 & 506 & 14 \\
\hline
\end{tabular}

$\mathrm{pH}^{\mathrm{exp}}$, the same as in Table 1.

a. Outlying first measurement/series rejected from the dataset according to Dixon's Q-test within $95 \%$ confidence.

using DPV at AgSA-PE. These peaks obviously correspond to successive irreversible reduction of individual nitro groups to the corresponding dihydroxyaminonaphthalenes. In the case of the formation of amino and/or hydrazine derivatives, voltammetric signals at more negative potential would appear. These results are comparable with the electrochemical behavior of the DNNs observed by DPV at a mercury meniscus modified silver solid amalgam electrode (m-AgSAE). ${ }^{17}$ The proposed mechanisms of DNN reduction at AgSA-PE result from polarographic studies at a dropping mercury electrode, coulometric experiments at a mercury pool electrode, and from further voltammetric measurements at a hanging mercury drop electrode (HMDE), ${ }^{15,16} \mathrm{~m}$-AgSAE,${ }^{17}$ and single-crystal silver amalgam electrodes. ${ }^{18}$ Poorly developed reduction signals of DNN in acidic media almost interfere with a signal provided by the reduction of silver ions at the beginning of the potential windows. These low reduction responses of the DNNs at AgSA-PE in strongly acidic media are obviously caused by a blockage of the phase boundary at the electrode surface by positively charged methanol and/or by hydrogen ions. Therefore, weakly acidic $(\mathrm{pH}>3)$ up to strongly alkaline media $(\mathrm{pH} 12.3)$ are recommended for voltammetric application of the AgSA-PE. Supporting electrolytes with $\mathrm{pH}^{\exp }$ 6.9, 12.3, and 8.7, were chosen for further investigation. From an analytical point of view, $\mathrm{pH}^{\exp } 12.3$ should be also selected for 1,3- and 1,8-DNN, but $\mathrm{pH}^{\exp }$ values of 6.9 and 8.7 , respectively, were chosen to compare the AgSA-PE's sensitivity for the DNNs at different $\mathrm{pH}$ values.

The repeatability of 18 consecutive measurements at one electrode surface without wiping of the paste (renewal of the electrode surface), and of a 7-times wiped electrode surface after 5 consecutive measurements was tested for all studied DNNs using DPV at AgSA-PE without any prior mechanical or electrochemical pretreatment in appropriate electrolytes. The evaluated peak heights were tested by Dixon's Q-test with $95 \%$ confidence, to reject the outlying measurements/series from the dataset, and then statistically evaluated (Table 2). The AgSA-PE provides good signal repeatability with an RSD of up to $5 \%$ ( $n=18$ or 17 of consecutive measurements at one electrode surface) and acceptable repeatability with RSD around $20 \%$ ( $n=35$ or 30 of five consecutive measurements at the 7 or 6 times wiped amalgam paste). The differences between the obtained RSDs are caused by a non-uniform particle distribution
Table 3 Parameters of the calibration straight lines with evaluated limits of detection of for DPV determination of DNNs at AgSA-PE in BR buffer-methanol (1:1) with appropriate $\mathrm{pH}^{\text {exp }}$

\begin{tabular}{ccccccc}
\hline $\begin{array}{c}\text { Compound } \\
\text { (BR-methanol) }\end{array}$ & Peak $\begin{array}{c}c l \\
\mu \mathrm{mol} \mathrm{l}^{-1}\end{array}$ & $\begin{array}{c}\text { Slope/ } \\
\mathrm{nA} \mu \mathrm{mol}^{-1}\end{array}$ & $\begin{array}{c}\text { Intercept/ } \\
\mathrm{nA}\end{array}$ & $\begin{array}{c}r^{2} \\
\mu \mathrm{mol} \mathrm{l}^{-1}\end{array}$ \\
\hline 1,3-DNN & 1 & $20-100$ & $-53.5 \pm 0.9$ & $111 \pm 60$ & 0.9988 & - \\
$\left(\mathrm{pH}^{\exp }\right.$ 6.9) & 2 & $20-100$ & $-35.0 \pm 1.5$ & $138 \pm 89$ & 0.9941 & - \\
& 1 & $1-10$ & $-42.0 \pm 1.9$ & $36 \pm 11$ & 0.9901 & 1 \\
& 2 & $1-10$ & $-17.8 \pm 0.6$ & $13.4 \pm 3.4$ & 0.9950 & 3 \\
$1,5-\mathrm{DNN}$ & 1 & $20-100$ & $-58.7 \pm 1.6$ & $61 \pm 47$ & 0.9812 & - \\
$\left(\mathrm{pH}^{\exp } 12.3\right)$ & 2 & $20-100$ & $-44.2 \pm 1.4$ & $-27 \pm 15$ & 0.9962 & - \\
& 1 & $1-10$ & $-33.9 \pm 1.6$ & $17.7 \pm 9.6$ & 0.9893 & 1 \\
& 2 & $1-10$ & $-21.5 \pm 0.8$ & $-2.4 \pm 4.9$ & 0.9930 & 2 \\
$1,8-\mathrm{DNN}$ & 1 & $20-100$ & $-27.6 \pm 1.3$ & $-248 \pm 82$ & 0.9921 & - \\
$\left(\mathrm{pH}{ }^{\exp } 8.7\right)$ & 1 & $1-10$ & $-39.1 \pm 1.1$ & $-14.4 \pm 6.8$ & 0.9960 & 1 \\
\hline
\end{tabular}

$\mathrm{pH}^{\text {exp }}$, the same as in Table 1 .

of the silver amalgam powder, causing worse repeatable electrode surface morphology after each wiping of the paste than in the case of CPEs based on uniformly sized carbon micro-beads (e.g., o.d. $5 \mu \mathrm{m}$ ). An attempt to increase the uniformity of the amalgam powder was not successful, because no suitable separation process or sieve with so small a porosity (up to $25 \mu \mathrm{m}$ ) was found. The preparation of silver amalgam powder using a reduction of mercury by silver nitrate with vigorous stirring was also tested, but no useful results were obtained, because of the formation of mercury salts during the reaction, which further affects the dissolution of the Paraffin paste during voltammetric measurements.

The sensitivities of the developed analytical methods for the determination of DNNs by DPV at AgSA-PE were evaluated from the corresponding calibration curves constructed in the ranges of $1-10$ (see Fig. 2) and $10-100 \mu \mathrm{mol} \mathrm{l}^{-1}$ in appropriate BR buffer-methanol mixtures (1:1). The resulting limits of detection $\left(L_{\mathrm{D}} \mathrm{S}\right)$ were calculated from standard deviations (SD) of 7 consecutive determinations of appropriate DNN $\left(c=1 \mu \mathrm{mol} \mathrm{l}^{-1}\right)$ and slopes of the corresponding calibration straight lines in the range of $1-10 \mu \mathrm{mol} \mathrm{l}^{-1}$, according to:

$$
L_{\mathrm{D}}=\frac{3 \times \mathrm{SD}}{\text { slope }}
$$

All of the relevant data are summarized in Table 3. The observed $L_{\mathrm{D}} \mathrm{S}$ around $1 \mu \mathrm{mol} \mathrm{1^{-1 }}$ were found to be comparable with the values provided by differential pulse polarography at DME. In spite of the fact that DNNs were determined by adsorptive stripping voltammetry at HMDE with an $L_{\mathrm{D}} \mathrm{S}$ of around $0.02 \mu \mathrm{mol} \mathrm{l}^{-1}$ in aqueous media, ${ }^{20}$ an attempt to decrease $L_{\mathrm{D}} \mathrm{S}$ using adsorptive stripping differential pulse voltammetry at AgSA-PE in aqueous medium without methanol was not successful. The results obtained with AgSA-PE were comparable to those obtained using DPV at $\mathrm{m}-\mathrm{AgSAE},{ }^{17}$ and at a single crystal silver amalgam electrode. ${ }^{18}$ All of these novel working electrodes offer specific advantages, such as simple renewal of the electrode surface, or the possibility of easy chemical modification. An easily renewable electrode surface of the AgSA-PE, which can be easily removed in the case of its passivation by strongly adsorbed interferents, or by products of electrode reactions, is the first big advantage of this novel electrode. A possibility of simple direct modification of the paste by proper additives is another advantage, which will be the subject of further investigation. 


\section{Conclusions}

It was proved that novel AgSA-PE is applicable for the voltammetric determination of organic nitro-compounds in aqueous-methanolic media (1:1), sensitive voltammetric methods for determination of the model compounds (1,3-, 1,5-, and 1,8-dinitronaphthalene) were developed. A simply renewable electrode surface of the AgSA-PE provides sufficient repeatability of the consecutive measurements (RSD around $20 \%$ with and $5 \%$ without paste wiping and/or any necessary electrochemical pretreatment or regeneration). Together with an easily renewable electrode surface and sufficient sensitivity of the AgSA-PE, it is possible to modify the paste by a proper choice of the pasting liquid, or other additives, which may further increase the sensitivity or selectivity of the sensor.

\section{Acknowledgements}

Financial support from Ministry of Education, Youth and Sports of the Czech Republic (Project MSM 0021620857 and KONTAKT (AMVIS) Project ME 10004), Grant Agency of the Czech Republic (Project P206/12/G151), Grant Agency of Charles University in Prague (Project 89710/2011/B-Ch/PrF), Charles University in Prague (Projects UNCE 2012/44 and SVV 2012265201), and Technology Agency of the Czech Republic (Project TA01020565) is gratefully acknowledged.

\section{References}

1. V. Vyskocil and J. Barek, Crit. Rev. Anal. Chem., 2009, 39, 173.

2. M. Fojta, Collect. Czech. Chem. Commun., 2004, 69, 715.
3. M. Fojta, F. Jelen, L. Havran, and E. Palecek, Curr. Anal. Chem., 2008, 4, 250.

4. J. Barek, J. Fischer, T. Navratil, K. Peckova, B. Yosypchuk, and J. Zima, Electroanalysis, 2007, 19, 2003.

5. T. Navratil and J. Barek, Crit. Rev. Anal. Chem., 2009, 39, 131.

6. K. Peckova, J. Musilova, and J. Barek, Crit. Rev. Anal. Chem., 2009, 39, 148.

7. I. Svancara, A. Walcarius, K. Kalcher, and K. Vytras, Cent. Eur. J. Chem., 2009, 7, 598.

8. I. Svancara, K. Vytras, J. Barek, and J. Zima, Crit. Rev. Anal. Chem., 2001, 31, 311.

9. X. Q. Zhang, Q. Guo, and D. X. Cui, Sensors, 2009, 9, 1033.

10. J. Wang, Anal. Chem., 1995, 67, R487.

11. B. Yosypchuk and L. Novotny, Crit. Rev. Anal. Chem., 2002, 32, 141.

12. B. Yosypchuk and J. Barek, Crit. Rev. Anal. Chem., 2009, 39, 189.

13. A. Danhel and J. Barek, Curr. Org. Chem., 2011, 15, 2957.

14. A. Danhel, B. Yosypchuk, V. Vyskocil, J. Zima, and J. Barek, J. Electroanal. Chem., 2011, 65, 218.

15. K. Shanmugam, J. Barek, and J. Zima, Chem. Anal. [Warsaw], 2004, 49, 765.

16. K. Shanmugam, J. Barek, and J. Zima, Collect. Czech Chem. Commun., 2004, 69, 2021.

17. A. Danhel, K. Peckova, K. Cizek, J. Barek, J. Zima, B. Yosypchuk, and T. Navratil, Chem. Listy, 2007, 101, 144.

18. J. Tvrdikova, A. Danhel, J. Barek, and V. Vyskocil, Electrochim. Acta, 2011, published online (doi: 10.1016/ j.electacta.2011.09.074).

19. F. T. Pastor, H. Dejmkova, J. Zima, and J. Barek, Collect. Czech. Chem. Commun., 2011, 76, 383.

20. K. Shanmugam, Ph. D. Thesis, Charles University in Prague, 2004. 